\title{
Michel Foucault, Judith Butler, y los cuerpos e identidades críticas, subversivas y deconstructivas de la Intersexualidad
}

\section{Michel Foucault, Judith Butler and Intersexuality's critical, subversive and deconstructive bodies and identities}

\author{
Araceli GonZÁlez VÁZQUEZ
}

Universidad de Cantabria

RESUMEN. La Intersexualidad es considerada una herramienta de crítica, subversión y deconstrucción de las categorías sexo y género, singularmente desde posiciones ligadas al constructivismo social y muy nutridas por las retóricas postestructuralistas. La propuesta de este artículo es la de discutir la recepción del pensamiento de Michel Foucault en los estudios sobre la Intersexualidad, y explicar qué conceptos y cómo se incorporan, y qué ideas contribuyen a desestabilizar o reificar dentro de estos debates.

Palabras clave: Intersexualidad, Foucault, Butler, Género, Biopoder.

La idea de la intersexualidad se encuentra en el centro de un debate muy complejo y activo, y por tanto el primer concepto sometido a revisión es precisamente el concepto de intersexualidad. Los cuerpos intersexuales poseen simultáneamente y con distinta variabilidad características sexuales masculinas y femeninas, que son de tipo cromosómico y fenotípico. La Medicina, en su diagnóstico de estos cuerpos, habla de diversas condiciones o sindromes y emplea diferentes formas para denominarlas, formas presentes en la actualidad en muchos otros discursos
ABSTRACT. Intersexuality is considered a critical, subversive and deconstructive tool for the categories of sex and gender, in particular from social constructivist theoretical positions well nurtured by post-estructuralist rethoric. The purpose of this article is to discuss the reception of Foucault's thought in studies on Intersexuality, explain his concepts and how they are incorporated, and which ideas they contribute to destabilizing or reifying within the current debates.

Key words: Intersexuality, Foucault, Butler, Gender, Biopower.

distintos de los médicos: «hermafroditismo», «síndrome de insensibilidad a los andrógenos», «síndrome de Turner», «síndrome de Klinefelter», «hiperplasia suprarrenal congénita»,... La palabra designa realidades/posibilidades corpóreas e identidades, y por lo tanto no está vinculada únicamente a lo que entiende la Ciencia por sexo, sino también a lo que define una parte de la Ciencia Social y la Filosofía como género.

Los debates alrededor de la Intersexualidad intervienen activamente en la reconfiguración de algunos conceptos cen- 
trales de la Antropología ${ }^{1}$, y lo hacen principalmente desde el activismo, desde la Antropología del Género/Antropología Feminista y desde la Antropología/Estudios Queer ${ }^{2}$. La reconfiguración citada se extiende a las categorías - llamadas con frecuencia dicotomías, opuestos, binomios, binarios, dimensiones, distinciones o sistemas - sexo/género, hombre/mujer, heterosexualidad/homosexualidad, y naturaleza/cultura. La Intersexualidad suele ser considerada como una herramienta de crítica, subversión y deconstrucción de las categorías expresadas, singularmente desde posiciones ligadas al constructivismo social y muy nutridas por las retóricas postestructuralistas en oposición al determinismo y al esencialismo, singularmente al biológico o biologicista.

Las investigaciones que se inscriben en este marco de estudio de la Intersexualidad también participan de otros debates intelectuales. La confluencia de temas y perspectivas de análisis es evidente. Los textos se enmarcan en el estudio de la Identidad, del Cuerpo y de la Sexualidad, $\mathrm{y}$ constituyen con frecuencia una fuerte crítica de los discursos médicos, tecnológicos y científicos, ya que remiten a la problematización de la gestión biomédica de lo corporal, lo sexual y lo identitario. La mayor parte de los autores que analizan esta cuestión desde un punto de vista político plantean la extensión de los Derechos Humanos y de las prácticas de libertad relativas a la identidad, al género y a la sexualidad. En ocasiones, los investigadores contribuyen a la articulación política y filosófica de las reivindicaciones y propuestas de los movimientos sociales e identitarios de sexo y de género, pero con mayor frecuencia reflexión y acción interactúan. La Intersexualidad es una cuestión que nos habla en última instancia sobre los procesos de subjetivización e individualización en la Postmodernidad, y por lo tanto nos sitúa en el fértil movimiento de reacción post-ilustrado. Lo que en definitiva está sometido a debate es la construcción moderna e ilustrada del sujeto y de los discursos sociales.

La incorporación de los estudios sobre la Intersexualidad en el seno de las antropologías anteriormente mencionadas es bastante irregular, aunque la bibliografía presenta en fechas recientes y con bastante insistencia un escenario de crisis de las categorías sexo/género que tiene sus deudas con estos estudios: así, y significativamente, Dorlin (2002) habla de «autopsia del sexo», Kessler (1997) propone la «disolución de la categoría género», y Stolcke titula provocadoramente uno de sus artículos, publicado en tres ocasiones (2003, 2004 y 2006), «la mujer es puro cuento». La idea de crisis de los conceptos es la que nos permite caracterizar con mayor fuerza el potencial crítico y subversivo de la Intersexualidad, al menos en un plano intelectual.

La Intersexualidad se construye en los discursos críticos básicamente como un gran desafío a la heteronormatividad (Kessler, 1997; Wiegman, 2006), al status normativo de la heterosexualidad. La producción bibliográfica de corte crítico en torno a la Intersexualidad hunde buena parte de sus raíces teóricas en las obras de Michel Foucault y Judith Butler. Las teorías llamadas postestructuralistas son bien conocidas y han tenido una gran influencia en los estudios encaminados a problematizar el género y lo intersexual, así como en general en buena parte de los estudios que constituyen el núcleo de la Antropología del Género; singularmente, han dado lugar a la teoría Queer, que no podría entenderse sin apelar a Foucault, a Derrida o a Lacan. La propuesta de este artículo es la de discutir la recepción del pensamiento de Michel Foucault y Judith Butler en los estudios sobre la Intersexualidad, desde las monografías de la 
bióloga Anne Fausto-Sterling a las tesis doctorales de los investigadores más jóvenes, pasando por otro tipo de publicaciones de las realmente escasas que existen sobre estas cuestiones. En última instancia, nuestra idea es la de explicar qué conceptos y cómo se incorporan, y qué ideas contribuyen a desestabilizar o reificar dentro de estos debates.

\section{Michel Foucault entre las feministas y sobre la Intersexualidad: Judith Butler lee a Foucault y habla sobre Herculine/Alexina/Abel}

Las obras de Michel Foucault han ejercido una notable influencia dentro de la Antropología del Género/Antropología Feminista (Martin, 1982; Butler, 1986; Sawicki, 1986; Diamond y Quinby, 1988; Alcoff, 1990; Sawicki, 1991; McNay, 1992; Dean, 1994; Hekman, 1996; Romero, 1996; McLaren, 2002; Oksala, 2004; Taylor y Vintges, 2004; Amigot, 2006). La recepción y reformulación teórica del pensamiento de Foucault ha dado lugar también a una interesante literatura sobre los límites de su discurso en relación con el Feminismo y sus principales temas (Alcoff, 1990; Bartky, 1990; Hartsock, 1990; Aladjem, 1991; Ramazanoglu, 1993; Deveaux, 1994, 1999; Rozmarin, 2005). La recepción de Foucault dentro de los Estudios Queer (Halperin, 1995; Spargo, 1999; Rousseau, 2000), y singularmente dentro de los Estudios sobre la Intersexualidad, le debe mucho a la obra de Judith Butler (Butler, 1986a, 1986b, 1989, 1992, 1995, 2000). La bibliografía incluye en los últimos años una buena nómina de análisis de la lectura de Foucault hecha por Butler y sobre los puntos de coincidencia y disensión de ambos teóricos (Weberman, 2000; Smith, 2001; Mills, $2003^{3}$; Dudrick, 2005).

Foucault escribió sobre lo intersexual, y lo hizo en una fecha tan temprana como $1978^{4}$, en la edición francesa del diario de Herculine Barbin, un hermafrodita francés de la segunda mitad del siglo XIX, y también en 1980, en su edición norteamericana de estas mismas memorias. Foucault presenta la historia de Herculine como «la prueba de la posibilidad de estados de sexualidad inclasificables, indeterminados en su género y por añadidura felices» (Moro Abadía, 2006: 167). La exigencia de identificación se ejercería con fuerza en este caso porque «no hay discurso para las personas sin verdadero sexo» (Moro Abadía, 2006: 168). La exigencia de identificación no procede del discurso médico, sino del Derecho, que «recogiendo las influencias de la ciencia médica, funcionará aquí como una práctica social fundamental a la hora de configurar formas de subjetividad y de exigir una estricta identificación sexual, un sexo verdadero y único, que aclare el estado civil del individuo») (Moro Abadía, 2006: 168).

La recepción de la obra de Foucault en la de Judith Butler es compleja, y no sólo por la oscura retórica de la filósofa norteamericana. Judith Butler comenta ampliamente las ideas de Foucault sobre el caso de Herculine Barbin en su obra Gender Trouble ${ }^{5}$. Butler elabora una crítica bastante directa de lo que juzga una representación «romántica» de la sexualidad de Barbin por parte del Foucault (Butler, 1989: 120). Foucault, al hablar del limbo feliz de la no-identidad en el que se encuentra Herculine en la etapa previa a la exigencia de una identidad sexual, entra en contradicción consigo mismo, especialmente con lo publicado en su Historia de la Sexualidad sobre la producción del sexo por parte de las complejas interacciones entre discurso y poder (Butler, 1989: 123).

Foucault también se refiere al caso de Anne Grandjean, juzgada en París en 1765. Los registros de la época ilustran la obligación jurídica y médica de elegir un 
sexo; en este caso, se opta por el que «predomina» en su anatomía, pero sobre todo se incide en la idea de que Grandjean ha de practicar las relaciones sexuales correspondientes a este sexo. Lo monstruoso en este caso no es su «hermafroditismo», sino su «lesbianismo», dado que tenía relaciones con una mujer (Cleminson y Medina Doménech, 2004). La sexualidad es un elemento importante en los dos casos; igualmente, Herculine Barbin tenía relaciones con una chica llamada Sara, con la que incluso albergaba la esperanza de casarse una vez se le había inscrito en el registro civil como hombre (Dreger, 2000: 18). La exigencia médico-legal de un sexo va unida a la exigencia de una determinada sexualidad.

Alice Domurat Dreger (1988) también ha comentado el análisis de Foucault, esta vez en una obra inserta plenamente en el debate de la Intersexualidad, la titulada Hermaphrodites and the medical invention of sex. La obra de Dreger le ha prestado similar atención a la intersexualidad en época histórica y en el presente. Como ella misma explica, en relación con su trayectoria investigadora, «it would have been obnoxious to write about the history of intersex without listening to what intersexed people thought about my ideas» (Domurat Dreger, 2000: 164). Dreger comenta el caso de Barbin desde otra perspectiva, y de centra en un principio en la muerte de Herculine, y por tanto en la figura de Abel Barbin. Los nombres que recibe esta persona a lo largo de su vida son, como vemos, varios: Adélaïde Herculine en su nacimiento; Alexina durante su infancia y juventud como apelativo familiar; y Abel durante sus últimos ocho años de vida (se suicida a los veintinueve años) (Dreger, 2000: 17-18). Le considera un ejemplo de la obsesión decimonónica por el sexo y la identidad sexual «verdaderas», y en este sentido su interpretación no difiere de la de Foucault.

\section{La recepción de Foucault en los estudios sobre la Intersexualidad}

La gestión biomédica de la Intersexualidad es una cuestión que ha sido ampliamente discutida en los últimos diez años, con 1998 como año de mayor producción bibliográfica. La crítica le debe mucho a la obra de la activista norteamericana Cheryl Chase, y a los estudios publicados por Fausto-Sterling, Kessler (1990, 1997), Holmes (1994, 2000) y Dreger (1998, 2000).

Los cuerpos intersexuales han sido denominados en la bibliografía «cuerpos dóciles»(corps dociles), «cuerpos ficticios» (fictitious bodies) y «cuerpos que engañan» (deceiving bodies) (Escabí Montalvo, 2006). En este artículo prefiero recuperar la noción de Butler de cuerpo subversivo. La idea de los cuerpos dóciles es de origen foucaultiano, y la desarrolla en su obra Vigilar y Castigar (1975), donde designa con esta noción a los cuerpos sometidos, moldeados por el poder disciplinario. La noción «cuerpos ficticios» (fictitious bodies) es hija del concepto de «sexo ficticio» (fictive sex) de Monique Wittig (muy difundido en la reapropiación de Butler, 1990), pero también encuentra su raíz en las obras de Hobbes y de Bentham, de donde deviene útil en el ámbito de la Política: también existen paralelos menos sociales en el ámbito de la Astronomía, donde un cuerpo ficticio es el que tiene masa cero $y$ momento de inercia. La subversión de la que habla Butler es performativa, es la de las acciones. En particular, las acciones del cuerpo subversivo son las que conducen a la problematización del Género, al Gender Trouble. La idea del cuerpo, por lo tanto, deviene central. 
La noción focaultiana de biopoder en la crítica a la gestión biomédica del cuerpo intersexual

Los estudios sobre la Intersexualidad, especialmente los más críticos con la Biomedicina están cuajados de retórica foucaultiana. Langlo se pregunta, como ya hiciera Foucault (1980), si verdaderamente necesitamos un sexo verdadero (Langlo, 1999: 13). Eckert habla de disciplinamiento (Eckert, 2006: 11), tal y como lo define Foucault en Vigilar y castigar, y utiliza las nociones normal y desviado definidas en la Historia de la Sexualidad. Mauro Cabral cita al Foucault de Las palabras y las cosas para sostener que es el establecimiento artificial de órdenes taxonómicos el que permite segregar las alteridades (Cabral, 2006: 5), en este caso «la alteridad intersexual». Gallacher se hace eco de la frase de Foucault que se refería a «la gestión de los cuerpos perversos del hermafrodita y el homo» (Gallacher, 2005: 9).

La noción de biopoder ha sido probablemente la de mayor éxito entre todas las que ha ido incorporando la literatura sobre la Intersexualidad. El concepto no llega a través de Butler, sino principalmente a través de la obra más influyente de la bióloga Anne Fausto-Sterling, Sexing the body (2000: 7-8). La incursión de Fausto-Sterling en el ámbito de la Intersexualidad culmina con el capítulo titulado: "Should there be only two sexes?». En la obra de Foucault, el biopoder se habría desarrollado en la época clásica «bajo dos formas principales relacionadas con la inscripción del poder en los cuerpos», la segunda de las cuáles, relacionada con las disciplinas del cuerpo, habría tenido por objeto «producir un ser humano que pueda ser tratado como un cuerpo dócil» (Moro Abadía, 2006: 129, Dreyfus y Rabinow, 1982: 197, citado en Moro, 2006). El biopoder, definido en la
Historia de la Sexualidad, también habría sido «un elemento indispensable en el desarrollo del capitalismo», que se habría afirmado a través de la «inserción controlada de los cuerpos en el aparato de producción y mediante un ajuste de los fenómenos de población a los procesos económicos» (Foucault, 170). La noción la incorpora en primera instancia Anne Fausto-Sterling en su controvertido ensayo The five sexes. Fausto-Sterling escribe lo siguiente:

«el tratamiento de la intersexualidad en este siglo proporciona un claro ejemplo de lo que el historiador francés Michel Foucault ha denominado biopoder. El conocimiento desarrollado en la bioquímica, la embriología, la endocrinología, la psicología y la cirugía ha permitido a los médicos controlar el sexo verdadero del cuerpo humano» (Fausto-Sterling, 1993, citada en Morland, 2005: 94).

Langlo (1999: 76) entiende que los tratamientos médicos aplicados a los cuerpos intersexuales, e incluso la percepción misma de que es necesario un tratamiento médico, son instancias del biopoder definido por Foucault. Eckert (2003: 37) subraya que la institucionalización del biopoder es una especie de producto de la extensión del determinismo biológico. La interpretación de Eckert es directamente deudora de la de Fausto-Sterling; «la naturalización del hermafrodita se habría producido como consecuencia de la extensión del biopoder» (Eckert, 2003: 38). La misma autora, Eckert (2006), introduce una variante en apariencia geográfica, pero completamente política, cuando califica este biopoder descrito por Foucault como «biopoder Occidental». Danielsson (2005: 24) se hace eco de la idea de inscripción en los cuerpos cuando identifica en ciertos actos y formas de pensar la existencia de una fuerza social lo suficientemente poderosa como para «grabar literalmente sus demandas dimórficas en la carne hu- 
mana», y la equipara con el biopoder foucaultiano, del que todos, señala, somos agentes. La «fuerza social» de Danielsson (2005) es «urgencia social por la heterosexualidad» en expresión de Adkins (1999). Leslie Gallacher concreta y define la reasignación de sexo y de género como un ejemplo palpable de biopoder (Gallacher, 2005: 9); el conocimiento generado por los discursos médicos permite el control del sexo por parte de los médicos. Gallacher cita a Fausto-Sterling y también reconoce su deuda. Gregori Flor (2006: 103), una de las pocas investigadoras españolas que trabaja sobre la Intersexualidad desde las perspectivas que aquí son objeto de análisis, también caracteriza como biopoder las prácticas de sometimiento del cuerpo ejercidas por la institución médica; el cuerpo del intersexual estaría sometido al biopoder incluso antes del nacimiento, cuando es observado y clasificado a través de las técnicas de diagnóstico prenatal.

Langlo es uno de los pocos investigadores que reconoce explícitamente la influencia de Foucault en su metodología de trabajo, no en vano se plantea el análisis de los discursos sobre la Intersexualidad. Lo que más sorprende en la recepción de Foucault en la bibliografía de la Intersexualidad es, por un lado, la falta de una verdadera integración de su discurso en los estudios (en la mayor parte de las ocasiones, tan sólo se le cita de forma erudita), y por otro, la escasa o casi nula problematización del discurso del propio Foucault en relación con el tema de estudio. La última cuestión es un hecho algo insólito, ya que a pesar de la fertilidad y la consistencia de su pensamiento, nos encontramos a veinticinco años de su muerte, y quizá no sólo por el paso del tiempo, sino por la intensidad de los procesos de transformación social vividos desde mediados de los ochenta, cabría esperar que las puntualizaciones hubieran sido algo más frecuentes y densas. En otro orden de cosas, siendo éste de la Intersexualidad un área de investigación en el que, como parece ser, las ideas de Foucault adquieren una alta significación, igualmente podría haber ocurrido que fuera esta la arena en la que ver más debate. Lamentablemente, la lectura de la bibliografía sugiere que no es así.

\section{Conclusiones}

La fuerza deconstructiva de las ideas de Michel Foucault ha contribuido de forma decisiva a fertilizar el debate de la Intersexualidad y desde él, a activar la problematización de las categorías sexo/género, homosexualidad/heterosexualidad, hombre/mujer y naturaleza/cultura. La recepción de la obra de Foucault por parte de Butler, la difusión de la obra de esta filósofa norteamericana dentro de la Antropología del Género, y la influencia de la performatividad han sido decisivas.

La retórica de la verdad (sexo verdadero), de la ficción (cuerpos ficticios), de la invención (invention of sex), del engaño (cuerpos que engañan) es casi omnipresente, por un lado para remitir a la idea de ambigüedad y de creatividad (idea de posibilidad, en definitiva), y por otro para incidir en la lastrante/castrante exigencia política, social y cultural de una verdad en distintos planos. Las ideas de Foucault y de Butler se complementan en este debate. Por un lado, Foucault sirve a la crítica de la gestión biomédica de la Intersexualidad a través de sus ideas sobre las estrategias del conocimiento y del poder. Por otro lado, la noción de performance de Butler sirve a la comprensión de una identidad de género en movimiento. La mayor parte de los estudiosos manifiestan una confianza absoluta en la productividad del pensamiento foucaultiano, expresada manifiestamente por Aladjem (1991: 289) cuando escribe que la visión de Foucault «reveals the com- 
plexity of the practices of the past in a way that may help us to construct practices of freedom (el subrayado es mío) with a similar regard for the richness and diversity of the present». Luego de las teorías, las críticas. ¿Es necesario reunir las críticas a la performatividad (Digeser, 1994; Boucher, 2006), y hablar ya, como parece que sugiere Beatriz Preciado, de inflación performativa?

La literatura académica continúa sin analizar en profundidad las propias prácticas de poder y formas de dominio inherentes a los discursos críticos, subversivos y deconstructores, en parte por la fascinación que despiertan. La crítica de la gestión biomédica de los cuerpos intersexuales puede hacer un hueco dentro del debate a otros temas igualmente controvertidos, más cercanos a lo político. $\mathrm{La}$ acción política en relación con la Intersexualidad es posible y necesaria, indudablemente, ya sea con la instrumentación de Foucault o Butler, o de cualquiera otra filosofía cuya influencia, vista la satisfacción general con el Foucault más incisivo, parece aún lejana.

\section{BIBLIOGRAFÍA}

Adkins, R. (1999): "Where "sex" is born(e): Intersexed births and the social urgency of Heterosexuality», Journal of medical humanities, vol. 20, n. ${ }^{\circ}$ : 117-133.

Aladjem, T. K. (1991): «The philosopher's prism: Foucault, Feminism and critique», Political Theory, n. ${ }^{\circ}$ 19: 277-291.

Alcoff, L. (1990): «Feminist politics and Foucault: The limits to a collaboration», en Dallery, A. y C. Scott (eds.): Crises in continental philosophy, Albany, SUNY Press.

Amigot Leache, P. y M. Pujal i Lombart (2006): «Ariadna danza: Lecturas fe- ministas de Foucault», Athenea digital, vol. 9: 100-130.

Bartky, S. (1990): Femininity and domination: Studies in the phenomenology of oppression, New York, Routledge.

Boucher, G. (2006): «The politics of performativity: A critique of Judith Butler», Parrhesia, n. ${ }^{\circ}$ 1: 112-141.

Butler, J. (1986a): «Sex and gender in Simone de Beauvoir's Second sex», Yale French studies, vol. 72: 35-49.

- (1986b): «Variations on Sex and Gender: Beauvoir, Wittig, and Foucault», Praxis International, n. ${ }^{\circ}$ 5: 505-516 (en castellano, traducción de Ana Sánchez: Variaciones sobre sexo y género: Beauvoir, Wittig y Foucault, en Teoría feminista y teoría crítica. Ensayos sobre la politica de género en las sociedades de capitalismo tardío, Ediciones Alfons el Magnànim, Valencia, 1990).

- (1989): «Foucault and the Paradox of Bodily Inscriptions», Journal of Philosophy, vol. 86, n. ${ }^{\circ}$ 11: 601-607.

- (1992): «Sexual Inversions: Rereading the End of Foucault's History of Sexuality, Vol. I», en Stanton, D.C. (ed). Discourses of Sexuality: From Aristotle to AIDS, University of Michigan Press, Ann Arbor: 344-361.

- (1995): «Subjection, Resistance, Resignification: Between Freud and Foucault», en J. Rajchman (ed). The Identity in Question, Routledge, London, New York: 229-249.

- (2000): «Subjection, Resistance, Resignification: Between Freud and Foucault», en W. Brogan, J. Risser (eds), American Continental Philosophy. Studies in Continental Thought, Indiana University Press, Bloomington, Indianápolis: 335-351.

Cabral, M. (2006): «Cuando digo intersex: un diálogo introductorio a la intersexualidad», Cuadernos Pagú, n. ${ }^{\circ} 24: 283-304$. 
Capurro, R. (2004): Del sexo y su sombra. Del misterioso hermafrodita de Michel Foucault, DF, Epelé.

Cleminson, R. y R. M. Medina Doménech (2004): «¿Mujer u hombre? Hermafroditismo, tecnologías médicas e identificación del sexo en España, 1860-1925», Dynamis, n. ${ }^{\circ} 24:$ 53-91. www.ugr.es/ rosam/pdf/Hermafroditas.pdf

Danielsson, M. (2005): Intersexuality and its medical and social implications, Malmö Högskola.

Dean, C. J. (1994): «The productive hypothesis: Foucault, gender, and the History of sexuality», History and theory, vol. 33, n. ${ }^{\circ}$ 3: 271-296.

Deveaux, M. (1994): «Feminism and empowerment: A critical reading of Foucault», Feminist studies, vol. 20, n. ${ }^{\circ}$ 2: 223-247.

- (1999): «Feminism and empowerment: a critical reading of Foucault», en Hesse-Biber, S. (ed.): Feminist Approaches to Theory and Methodology: An Interdisciplinary Reader, Oxford University Press.

Diamond, I. (1988): «Feminism and Foucault: Reflections on resistance», en Diamond, I y L. Quinby (eds.): $F e$ minism and Foucault: Reflections on resistance, Boston, Northeastern University Press: XI-XIX.

Digeser, P. (1994): «Performativity trouble: Postmodern feminist and essential subjects», Political research quarterly, vol. 47, n. ${ }^{\circ}$ 3: 655-673.

Domurat Dreger, A. (2000): «Jarring bodies: thoughts on the display of unusual anatomies», Perspectives in biology and medicine, vol. 43, n. $^{\circ} 2$ : 161-172.

Dorlin, E. (2002): «Autopsie du sexe», Les temps modernes, . $^{\circ}$ 619: 115-143.

Dreger, A. D. (1988, 2000): Hermaphrodites and the medical invention of sex, Harvard University Press.
Dudrick, D. (2005): «Foucault, Butler, and the Body», European Journal of Philosophy, vol. 13, n. ${ }^{\circ}$ 2: 226-246.

Eckert, C. A. (2003): The historicisation of the hermaphroditic/Intersexed body: From medicalisation to de-medicalisation, MA Thesis, University of Sussex.

Eckert, L. (2006): «The third -A hindrance to diversity?», UK Postgraduate Conference in Gender Studies, 21-22 june. http:/www.leeds.ac.uk/ gender-studies/events/epapers/epaper 31\%20Lena $\% 20$ Eckert.pdf

Escabí-Montalvo, A. y J. Toro-Alfonso (2006): «Cuando los cuerpos engañan: un acercamiento crítico a la categoría de la intersexualidad», International Journal of Clinical and Health Psychology, vol. 6, n. ${ }^{\circ}$ 3: 753-772.

Fausto-Sterling, A. (1993): «The five sexes: Why male and female are not enough», The Sciences, 33.

- (2000): Sexing the body: Gender politics and the construction of sexuality, New York, Basic Books.

Foucault, M. (1978a): «Il misterioso ermafrodito», en Dits et écrits II: 1976-1988, Gallimard, Paris, 2001: 624-625.

- (1978b): Herculine Barbin dite Alexina B., Gallimard, Paris.

— (1980a): «Herculine Barbin: Being the recently discovered memoirs of a nineteenth-century French hermaphrodite». Brighton, Harvester Press.

- (1980b): «Le vrai sexe», en Dits et écrits II: 1976-1988. Gallimard, Paris, 2001: 934-942.

- (1980c): Herculine Barbin: Being the recently discovered memoirs of a nineteenth century French hermaphrodite. New York, Pantheon.

- (1985): Herculine Barbin llamada Alexine B. Madrid, Revolución.

Fox, N. J. (1998): «Foucault, foucauldians and sociology», The British 
journal of sociology, vol. 49, n. ${ }^{\circ} 3$ : 415-433.

Gallacher, L. (2005): The psychology of Intersex: Research into the experiences of individuals/parents who have experienced androgen insensitivity syndrome or congenital adrenal hyperplasia within the UK, MA Dissertation, University of York.

Gregori Flor, N. (2006): «Los cuerpos ficticios de la biomedicina. El proceso de construcción del género en los protocolos médicos de asignación de sexo en bebés intersexuales», AIBR, Revista de Antropología iberoamericana, vol. 1, n. ${ }^{\circ}$ 1: 103-124.

Halperin, D. (1995): Saint Foucault: Towards a Gay hagiography. New York, Oxford University Press.

Hartsock, N. (1990): «Foucault on power: A theory for women?», en Nicholson, L. (ed.): Feminism/Postmodernism. New York, Routledge.

Hekman, S. J. (ed.; 1996): Feminist interpretations of Michel Foucault. Pennsylvania University Press.

Holmes, M. M. (1994): Medical politics and cultural imperatives: intersexual identities beyond pathology and erasure. York University, Canada. MA Thesis.

- (2000): The doctor will fix everything: intersexuality in contemporary culture, Concordia University, Canada, $\mathrm{PhD}$ Thesis.

Kessler, S. J. (1990): «The medical construction of gender: case management of intersexed infants», Signs, 16, n. ${ }^{\circ} 1: 3-26$.

— (1997): «Creating good-looking genitals in the service of gender», en $\mathrm{M}$. Duberman (ed.) A queer world: The Centre for Lesbian and Gay Studies reader, New York, New York University Press: 153-173.

Lafrance, M. (2002): «Uncertain erotic: A Foucauldian reading of Herculine
Barbin dite Alexina B.», Sites: The Journal of Contemporary French Studies, vol. 6, n. ${ }^{\circ}$ 1: 119-131.

Langlo, L. (1999): Intertextuality about Intersexuality, MA Thesis, University of Amsterdam.

Martin, B. (1982): «Feminism, criticism and Foucault», New German critique, n. ${ }^{\circ}$ 27: 3-30.

Martín Casares, A. (2006): Antropología del género. Culturas, mitos y estereotipos sexuales, Ediciones Cátedra.

McLaren, M. (2002): Feminism, Foucault and Embodied subjectivity, Albany, SUNY Press.

McNay, L. (1992): Foucault and feminism: power, gender and the self, Cambridge, Polity Press.

Mills, C. (2003): «Contesting the political: Butler and Foucault on power and resistance», Journal of Political Philosophy, vol. 11, n. ${ }^{\circ}$ 3: 253-272.

Morland, I. (2005): Narrating intersex: On the ethical critique of the medical management of intersexuality (1985-2005), PhD Thesis, University of London.

Moro Abadía, O. (2006): La perspectiva genealógica de la historia, Universidad de Cantabria, Santander.

Oksala, J. (2004): «Anarchic bodies: Foucault and the Feminist question of experience», Hypatia, vol. 19, n. ${ }^{\circ} 4$ : 97-119.

Pelayo González-Torre, Á. y O. Moro Abadía (2003): «Michel Foucault y el problema del género», Doxa, n. ${ }^{\circ} 26$ : 847-869.

Ramazanoglu, C. (ed.; 1993): Up against Foucault: Explorations of some tensions between Foucault and feminism, Routledge UK, London.

Rousseau, G. S. (2000): «Foucault and the fortunes of Queer theory», The European legacy, vol. 5, n. ${ }^{\circ}$ 3: 401-413.

Rozmarin, M. (2005): «Power, freedom and individuality: Foucault and se- 
xual difference», Human studies, vol. 28, n. ${ }^{\circ}$ 1: 1-14.

Sawicki, J. (1986): «Foucault and Feminism: Towards a politics of difference», Hypatia, vol. 1: 23-26.

- (1991): Disciplining Foucault: Feminism, power and the body, Routledge UK, London.

Smith, A. M. (2001): «Missing Poststructuralism, Missing Foucault: Butler and Fraser on Capitalism and the Regulation of Sexuality», Social Text, vol. 19, n. ${ }^{\circ}$ 2: 103-125.

Spargo, T. (1999): Foucault and Queer theory. Icon Books, Ltd.

Stolcke, V. (2003): «La mujer es puro cuento: La cultura del género», Qua- derns de l'Institut Català d'Antropologia, n. ${ }^{\text {1 }}$ 19: 69-95.

- (2004): «La mujer es puro cuento: La cultura del género», Estudos feministas, Florianópolis, vol. 12, n. ${ }^{\circ}$ 2: 77-105.

- (2006): «La mujer es puro cuento: La cultura del género», Desarrollo económico, vol. 45, n. ${ }^{\circ}$ 180: 523-546.

Taylor, D. y K. Vintges (eds.; 2004): Feminism and the Final Foucault, University of Illinois Press.

Weberman, D. (2000): «Are freedom and anti-humanism compatible? The case of Foucault and Butler», Constellations, vol. 7, n. $^{\circ}$ 2: 255-271.

Wiegman, R. (2006): «Heteronormativity and the desire for gender», Feminist Theory, vol. 7, n. ${ }^{\circ}$ 1: 89-104.

\section{NOTAS}

1 La mayor parte de los estudios sobre la Intersexualidad publicados hasta la fecha son de carácter antropológico o médico-científico, y hablar de Antropología en este artículo no pretende oscurecer la existencia de una interesante reflexión filosófica en torno a este tema. Por el contrario, entre los propósitos de este artículo está el de analizar el anclaje filosófico de los debates activos en torno a la Intersexualidad.

${ }^{2}$ Lo queer haría referencia (Martín Casares, 2006 : 279) a: lesbianas, gays, bisexuales, transgénero, transexuales, homosexuales e intersexuales. La enumeración es útil desde un punto de vista heurístico, pero es necesario dejar constancia de que existen notables grados de disconformidad con la etiqueta queer dentro de los colectivos señalados.

${ }^{3}$ Catherine Mills ha trabajado sobre los puntos de fricción y encuentro entre Foucault y Butler en su tesis doctoral inédita: Mills, C. (2003), The Politics of Mere
Life: Foucault, Butler and Agamben on Biopolitics, Subjectivation and Violence, Unpublished $\mathrm{PhD}$ Thesis, Australian National University.

4 Foucault edita las memorias de Herculine Barbin en francés en 1978; existe una edición de la obra en inglés de 1980 y una edición en castellano de 1985. La introducción a la edición norteamericana de 1980, titulada «Le vrai sexe», ha sido incluida en la obra Dits et écrits, vol. II. Ver, sobre este caso analizado por Foucault: Lafrance, M. (2002): «Uncertain erotic: A Foucauldian reading of Herculine Barbin dite Alexina B.», Sites: The Journal of Contemporary French Studies, vol. 6, n. ${ }^{\circ}$ 1: 119-131; Capurro, R. (2004): Del sexo y su sombra. Del misterioso hermafrodita de Michel Foucault, DF, Epelé.

5 En concreto, en el epígrafe titulado «Foucault, Herculine, and the Politics of Sexual Discontinuity», Gender Trouble: 93-111. 